\title{
PROPORTIONAL RATIOS ORDERS BASED ON LAPLACE TRANSFORMS
}

\author{
JARRAHIFERIZ, J., MOHTASHAMI BORZADARAN, G. R.* AND REZAEI ROKNA- \\ BADI, A. H.
}

\begin{abstract}
The purpose of this paper is to generalize some stochastic orders based on the Laplace transform to proportional state and obtain some of their properties. Also, we discuss these generalized orders version in view of the weighted distributions.
\end{abstract}

Mathematics Subject Classification 2000: 62E10, 60E05, 60E15

Additional Key Words and Phrases: Laplace transform, Stochastic order, likelihood ratio order, (reversed) hazard rate order

\section{INTRODUCTION}

As an useful notion in applied mathematics and engineering, Laplace transform is also important in many areas of the probability and statistics (see Feller 1971). It is usually convenient to use Laplace transform rather than the characteristic function when the concerned random variable is non-negative. Several concepts of the stochastic comparisons between random variables have been defined and studied in the literature, since they are useful tools in many different areas of the applied probability and statistics (see Shaked and Shanthikumar 2007). Some of these comparisons are likelihood ratio order, proportional likelihood ratio order, Laplace transform order, Laplace transform ratio order, reversed Laplace transform ratio order and differentiated Laplace transform ratio order.

Rolski and Stoyan (1976) defined the Laplace transform order based on the Laplace transform. Then Klefsjo (1983) obtained a useful aging property of it and Alzaid et al. (1991) presented general preservation properties and applications of this order. Also, Shaked and Wong (1997) introduced stochastic orders based on the ratios of the Laplace transforms. Bartoszewicz (1999) studied characterizations of the stochastic orders based on the ratios of the Laplace transform. Bartoszewicz (2000) and Nanda (2000) achieved stochastic orders based on the Laplace transform and infinitely divisible distributions and generalized aging classes in terms of the Laplace transforms respectively. Recently, Li et al. (2009) introduced a new stochastic order based upon Laplace transform with applications.

Ramos-Romero and Sordo-Diaz (2001) generalized the likelihood ratio order called proportional likelihood ratio order to proportional state for absolutely continuous non-negative random variables and studied some properties and applications of it.

\footnotetext{
*Corresponding author.
} 
In this paper, we have presented some definitions of stochastic orders and some tools that are needed for expressing our results. Then, in sections 3 and 4 we have achieved (reversed) Laplace transform ratio and differentiated Laplace transform ratio orders respectively to proportional state and studied their properties and relations that theses results are generalized version of the previous cases. Finally, we have studied the proportional Laplace transform ordering in view of the weighted distributions.

\section{PRELIMINARIES}

Let $X$ and $Y$ be non-negative absolutely continuous random variables with density functions $f$ and $g$, distribution functions $F$ and $G$ and survival functions $\bar{F}$ and $\bar{G}$ respectively. Throughout the paper we take $a / 0$ to be $\infty$, whenever $a>0$. Let

$$
L_{X}(s)=E\left(e^{-s X}\right)=\int_{0}^{\infty} e^{-s x} f_{X}(x) d x
$$

denote the Laplace transform of $X$ which is a decreasing function in $s>0$. Consider the Laplace transform of $F$ as

$$
L_{X}^{*}(s)=\int_{0}^{\infty} e^{-s x} F_{X}(x) d x,
$$

and define the Laplace transform of $\bar{F}$ as

$$
L_{X}^{* *}(s)=\int_{0}^{\infty} e^{-s x} \bar{F}_{X}(x) d x .
$$

for all $s>0$. It is easy to verify $L_{X}^{*}(s)=\frac{1}{s} L_{X}(s)$ and $L_{X}^{* *}(s)=\frac{1-L_{X}(s)}{s}$. Also, $L_{Y}(s), L_{Y}^{*}(s)$ and $L_{Y}^{* *}(s)$ are defined for $Y$ like X.

More details related to Laplace Transforms and the interpretations corresponding to models in reliability, insurance, inventory and maintenance can be find in Alzaid et al. (1991) and Shaked and Wong (1997).

Here, we present some definitions which are used to present our main results.

Definition 2.1. $X$ is said to be smaller than $Y$ in the

(i) likelihood ratio order $\left(X \leq_{l r} Y\right)$, if $\frac{g(x)}{f(x)}$ increases in $x$ over the union of the supports of $X$ and $Y$.

(ii) Laplace transform order $\left(X \leq_{L t} Y\right)$, if $L_{X}(s) \geq L_{Y}(s) \forall s>0$.

(iii) Laplace transform ratio order $\left(X \leq_{L t-r} Y\right)$, if $\frac{L_{X}(s)}{L_{Y}(s)}$ is increasing in $s>0$.

(iv) reversed Laplace transform ratio order $\left(X \leq_{r-L t-r} Y\right)$, if $\frac{1-L_{X}(s)}{1-L_{Y}(s)}$ increases in $s>0$.

REMARK 2.2. For random variables $X$ and $Y$ we have

(1) $X \leq_{L t} Y \Leftrightarrow L_{X}(s) \geq L_{Y}(s) \Leftrightarrow L_{X}^{*}(s) \geq L_{Y}^{*}(s) \Leftrightarrow L_{X}^{* *}(s) \leq L_{Y}^{* *}(s)$. 
(2) $X \leq_{L t-r} Y\left(X \leq_{r-L t-r} Y\right) \Longleftrightarrow \frac{1-s L_{Y}^{* *}(s)}{1-s L_{X}^{* *}(s)}\left(\frac{L_{Y}^{* *}(s)}{L_{X}^{* *}(s)}\right)$ is decreasing in $s>0$.

Definition 2.3. A function $\phi:[0, \infty) \longrightarrow R$ is said to be completely monotone if all its derivatives $\phi^{(n)}$ exist and satisfy, $(-1)^{n} \phi^{(n)}(x) \geq 0$ for all $x>0$ and $n=0,1,2, \ldots$, where $\phi^{(n)}(x)$ denotes derivative of order $n$ of $\phi$.

Ramos-Romero and Sordo-Diaz (2001) and then Belzunce et al. (2002) studied likelihood ratio order and (reversed) hazard rate order to proportional state respectively. They obtained interesting properties and applications of them and Looked their relationships to other stochastic orders.

DeFinition 2.4. For non-negative absolutely continuous random variables $X$ and $Y$, we say that $X$ is smaller than $Y$ in the

(i) proportional likelihood ratio order $\left(X \leq_{p l r} Y\right)$, if $\frac{g(\lambda x)}{f(x)}$ increases in $x$ for any positive constant $\lambda<1$ over the union of the supports of $X$ and $Y$.

(ii) proportional hazard rate [reversed hazard rate] order $\left(X \leq_{p h r}\left[\leq_{p r h}\right] Y\right)$, if $\frac{\bar{G}(\lambda x)}{\bar{F}(x)}\left[\frac{G(\lambda x)}{F(x)}\right]$ increases in $x$ for any positive constant $\lambda<1$ over the union of the supports of $X$ and $Y$.

Now, in the next sections we will discuss our novelties.

\section{PROPORTIONAL AND REVERSED PROPORTIONAL LAPLACE TRANSFORM RATIO ORDER}

We can consider the Laplace transform as a mixture distribution function of exponential distribution with mean $\frac{1}{x}$ and a distribution with distribution function $F(\cdot)$. So it is interesting which we consider such orderings based on Laplace transform ratios and studied them for proportional state.

Definition 3.1. For non-negative absolutely continuous random variables $X$ and $Y$ we say that

(i) $X$ is smaller than $Y$ in the proportional Laplace transform ratio order $\left(X \leq_{p L t-r}\right.$ $Y)$, if $\frac{L_{Y}(\lambda s)}{L_{X}(s)}$ is decreasing in $s>0$, for any positive constant $\lambda<1$.

(ii) $X$ is smaller than $Y$ in the reversed proportional Laplace transform ratio order $\left(X \leq_{r-p L t-r} Y\right)$, if $\frac{1-L_{Y}(\lambda s)}{1-L_{X}(s)}$ is decreasing in $s>0$ for any positive constant $\lambda<1$.

Example1 Let $X \sim \operatorname{Exp}\left(\theta_{1}\right)$ and $Y \sim \operatorname{Exp}\left(\theta_{2}\right)$. If $\theta_{1}<\theta_{2}$, then $X \leq_{p l t-r} Y$ and if $\theta_{1}<\lambda \theta_{2}$ then $X \leq_{r-p l t-r} Y$.

It is clear that, $X \leq_{p L t-r} Y \Rightarrow X \leq_{L t-r} Y$ and $X \leq_{r-p L t-r} Y \Rightarrow X \leq_{r-L t-r} Y$ and there is not relationship between $\leq_{p L t-r}$ and $\leq_{r-p L t-r}$.

The first theorem below gives some necessary and sufficient technical conditions for (reversed) proportional Laplace transform ratio orders. We need to introduce 
JARRAHIFERIZ, J., MOHTASHAMI B. G. R., REZAEI R. A. H.

some notations. Let $Y$ be a non-negative absolutely continuous random variable. Denote

$$
\begin{gathered}
Q_{Y}(0, t)=g(t), \quad t \geq 0 \\
Q_{Y}(k, t)=\int_{0}^{t} Q_{Y}(k-1, x) d x, \quad t \geq 0, k=1,2, \ldots \\
\bar{Q}_{Y}(1, t)=\bar{G}(t), \quad t \geq 0
\end{gathered}
$$

and

$$
\bar{Q}_{Y}(k, t)=\int_{0}^{t} \bar{Q}_{Y}(k-1, x) d x, \quad t \geq 0, k=2,3, \ldots
$$

Similarly, for another non-negative random variable $X$ define, $Q_{X}(k, t)$ and $\bar{Q}_{X}(k, t)$. Also, for a function $\psi$ define

$$
\Delta^{0} \psi(n)=\psi(n), \quad \Delta^{k+1} \psi(n)=\Delta^{k} \psi(n)-\Delta^{k} \psi(n-1), \quad n \geq k+1, k=0,1,2, \ldots
$$

which is used in the following notes:

TheOREM 3.2. Let $X$ and $Y$ be non-negative absolutely continuous random variables.

a) $X \leq_{p L t-r} Y$ if and only if, $\frac{\int_{0}^{\infty} e^{-\lambda s t} Q_{Y}(k, t) d t}{\int_{0}^{\infty} e^{-s t} Q_{X}(k, t) d t}, \quad \forall k=0,1,2, \ldots$ is decreasing in $s>0$.

b) $X \leq_{r-p L t-r} Y$ if and only if, $\frac{\int_{0}^{\infty} e^{-\lambda s t} \bar{Q}_{Y}(k, t) d t}{\int_{0}^{\infty} e^{-s t} \bar{Q}_{X}(k, t) d t}, \quad \forall k=1,2, \ldots$ is decreasing in $s>0$.

Proof Using integration by parts, we have

$$
\begin{gathered}
L_{Y}(\lambda s)=\int_{0}^{\infty} \lambda^{k} s^{k} e^{-\lambda s t} Q_{Y}(k, t) d t, \\
1-L_{Y}(\lambda s)=\int_{0}^{\infty} \lambda^{k} s^{k} e^{-\lambda s t} \bar{Q}_{Y}(k, t) d t, \\
L_{X}(s)=\int_{0}^{\infty} s^{k} e^{-s t} Q_{X}(k, t) d t,
\end{gathered}
$$

and

$$
1-L_{X}(s)=\int_{0}^{\infty} s^{k} e^{-s t} \bar{Q}_{X}(k, t) d t,
$$

for $k=0,1, \ldots$. By $(8)$ and $(10)$ and part $(i)$ of Definition 3.1, we can prove $(a)$. Also, by (9) and (11) and part (ii) of Definition 3.1, we can prove (b). 
Definition 3.3. The function $f: A \times B \rightarrow[0, \infty)$ is $T P_{2}^{\dagger}\left(R R_{2}^{\ddagger}\right)$, if for all $a_{1} \leq a_{2}$ and $b_{1}<b_{2},\left(a_{i} \in A, b_{i} \in B, \quad i=1,2\right)$,

$$
\left|\begin{array}{ll}
f\left(a_{1}, b_{1}\right) & f\left(a_{1}, b_{2}\right) \\
f\left(a_{2}, b_{1}\right) & f\left(a_{2}, b_{2}\right)
\end{array}\right| \geq(\leq) 0
$$

Theorem 3.4. Let $X$ and $Y$ be non-negative absolutely continuous random variables. Then via the arguments in (4), (5), (6) and (7), the following assertions are hold:

a) If $\frac{Q_{Y}(k, t)}{Q_{X}(k, t)}, \quad \forall k \geq 0$ is increasing in $t$, then $X \leq_{p L t-r} Y$.

b) If $\frac{\bar{Q}_{Y}(k, t)}{\bar{Q}_{X}(k, t)}, \quad \forall k \geq 0$ is increasing in $t$, then $X \leq_{r-p L t-r} Y$.

Proof Let $Q_{X}(k, t)=Q_{1}(k, t)$ and $Q_{Y}(k, t)=Q_{2}(k, t)$. Since $\frac{Q_{Y}(k, t)}{Q_{X}(k, t)}, \quad \forall k \geq 0$ is increasing in $t$, then $\frac{Q_{2}\left(k, t_{2}\right)}{Q_{1}\left(k, t_{2}\right)} \geq \frac{Q_{2}\left(k, t_{1}\right)}{Q_{1}\left(k, t_{1}\right)}, t_{2}>t_{1}$, so $Q_{j}(k, t), \quad t>0, j \in\{1,2\}$ is $T P_{2}, e^{-s t}$ and $e^{-\lambda s t}$ are $R R_{2}$ in $s>0$ and $t \geq 0$ (Karlin 1968, Lemma 1.1, P.99). Then $\int_{0}^{\infty} e^{-s t} Q_{j}(k, t) d t$ and $\int_{0}^{\infty} e^{-\lambda s t} Q_{j}(k, t) d t$ are $R R_{2}$ for $j=1,2$, so, $\frac{\int_{0}^{\infty} e^{-\lambda s t} Q_{2}(k, t) d t}{\int_{0}^{\infty} e^{-s t} Q_{1}(k, t) d t}=\frac{\int_{0}^{\infty} e^{-\lambda s t} Q_{Y}(k, t) d t}{\int_{0}^{\infty} e^{-s t} Q_{X}(k, t) d t}$ is decreasing in $s>0$. Using Theorem 3.2 completed the proof. The proof of $(b)$ is similar.

REMARK 3.5. If in Theorem 3.4, take $\lambda=k=1$ then,

a) If $X \leq_{r h} Y$ (that is $\frac{G_{Y}(t)}{F_{X}(t)}$ be increasing in $t$ ), then $X \leq_{L t-r} Y$.

b) If $X \leq_{h r} Y$ (that is $\frac{\bar{G}_{Y}(t)}{F_{X}(t)}$ be increasing in $t$ ), then $X \leq_{r-L t-r} Y$.

THEOREM 3.6. Let $X$ and $Y$ be non-negative absolutely continuous random variables with corresponding moments $\nu_{i}, \quad \mu_{i}, \quad i=1,2, \ldots,\left(\mu_{0}=\nu_{0}=1\right)$ then,

a) $X \leq_{p L t-r} Y$ if and only if,

$$
\frac{\sum_{i=0}^{\infty} \frac{(-\lambda s)^{i}}{i !} \nu_{i}}{\sum_{i=0}^{\infty} \frac{(-s)^{i}}{i !} \mu_{i}}
$$

is decreasing in $s>0$.

b) $X \leq_{r-p L t-r} Y$ if and only if,

$$
\frac{\sum_{i=1}^{\infty} \frac{(-\lambda s)^{i}}{i !} \nu_{i}}{\sum_{i=1}^{\infty} \frac{(-s)^{i}}{i !} \mu_{i}}
$$

\footnotetext{
${ }^{\dagger}$ Totally Positive of Order2

${ }^{\ddagger}$ Reverse Regular Order2
} 
is decreasing in $s>0$.

Proof Via arguments in Theorem 5.B.2 of Shaked and Shanthikumar (2007) it is easily to seen that,

$$
\frac{L_{Y}(\lambda s)}{L_{X}(s)}=\frac{\sum_{i=0}^{\infty} \frac{(-\lambda s)^{i}}{i !} \nu_{i}}{\sum_{i=0}^{\infty} \frac{(-s)^{i}}{i !} \mu_{i}} .
$$

So, (a) follows from Definition 3.1 and the proof of $(b)$ is similar to $(a)$.

\section{PROPORTIONAL DIFFERENTIATED LAPLACE TRANSFORM RATIO ORDER}

Li et al. (2009) introduced a new stochastic order based on the Laplace transform with its applications and called it differentiated Laplace transform ratio ordering. First, we express this ordering for proportional state and then obtained some of its properties.

As we said the Laplace transform of the non-negative absolutely continuous random variable $X$ is given by $L_{X}(s)=\int_{0}^{\infty} e^{-s x} f_{X}(x) d x$. Hence,

$$
\Psi_{X}(s)=1-L_{X}(s)=\int_{0}^{\infty}\left(1-e^{-s u}\right) d F(u), \quad s>0,
$$

which can be considered as a mixture distribution of the exponential distribution with mean $\frac{1}{x},(x>0)$ and mixing distribution $F$. Thus, $\Psi$ is a distribution with a density $\psi_{X}(s)=\int_{0}^{\infty} x e^{-s x} f(x) d x, \quad s>0$. Suppose that the random variable with this distribution is denoted by $\zeta(X)$. Many properties and characterization of $\zeta(X)$ proved in Bartoszewicz (1999). For example, he proved that,

$$
X \leq_{L t(L t-r, r-L t-r)} Y \Leftrightarrow \zeta(Y) \leq_{s t(h r, r h)} \zeta(X) .
$$

Based on this view, Li et al. (2009) presented a new order and called it differentiated Laplace transform ratio order.

Definition 4.1. Suppose that $X$ and $Y$ be non-negative absolutely continuous random variables. We say that $X$ is smaller than $Y$ in the differentiated Laplace transform ratio order $\left(X \leq_{d-L t-r} Y\right)$ if $\frac{L_{Y}^{\prime}(s)}{L_{X}^{\prime}(s)}$ is decreasing in $s>0$, such that $-L_{X}^{\prime}(s)=\psi_{X}(s)$ and $-L_{Y}^{\prime}(s)=\psi_{Y}(s)$ are densities of $\zeta(X)$ and $\zeta(Y)$.

Remark 4.2. It is easily to seen that $X \leq_{d-L t-r} Y \Leftrightarrow \zeta(Y) \leq_{l r} \zeta(X)$. Also, $X \leq_{d-L t-r} Y$ implies that $X \leq_{L t-r} Y$ and $X \leq_{r-L t-r} Y$.

Definition 4.3. Suppose that $X$ and $Y$ be non-negative absolutely continuous random variables. We say $X$ is smaller than $Y$ in the proportional differentiated Laplace transform ratio order $\left(X \leq_{d-p L t-r} Y\right)$ if $\frac{L_{Y}^{\prime}(\lambda s)}{L_{X}^{\prime}(s)}$ is decreasing in $s>0$, such that $-L_{X}^{\prime}(s)=\psi_{X}(s)$ and $-L_{Y}^{\prime}(s)=\psi_{Y}(s)$ are density functions of $\zeta(X)$ and $\zeta(Y)$ respectively.

ThEOREM 4.4. Let $X_{1}, X_{2}, \ldots$ be iid non-negative absolutely continuous random variables and $N_{1}$ and $N_{2}$ be two positive integer-valued random variables that are 
independent of $X_{i}$ 's. Then, $N_{1} \leq_{d-p L t-r} N_{2} \Leftrightarrow \sum_{i=1}^{N_{1}} X_{i} \leq_{d-p L t-r} \sum_{i=1}^{N_{2}} X_{i}$.

Proof $X_{i}$ 's are iid so, $L_{X_{1}+\ldots+X_{N_{j}}}(\lambda s)=\sum_{i=1}^{\infty} P_{N_{j}}(i) L_{X_{1}}^{i}(\lambda s)$, then, on noting that in Theorem 5.B.3 of Shaked and Shanthikumar (2007)

$$
\begin{aligned}
\frac{\partial}{\partial s} L_{X_{1}+\ldots+X_{N_{j}}}(\lambda s) & =\frac{L_{X_{1}}^{\prime}(\lambda s)}{L_{X_{1}}(\lambda s)} \lambda \sum_{i=1}^{\infty} i P_{N_{j}}(i) e^{i \ln \left(L_{X_{1}}(\lambda s)\right)} \\
& =\frac{\partial}{\partial s} L_{N_{j}}\left(-\ln L_{X_{1}}(\lambda s)\right),
\end{aligned}
$$

hence,

$$
\frac{\frac{\partial}{\partial s} L_{X_{1}+\ldots+X_{N_{2}}}(\lambda s)}{\frac{d}{d s} L_{X_{1}+\ldots+X_{N_{1}}}(s)}=\frac{\frac{\partial}{\partial s} L_{N_{2}}\left(-\ln L_{X_{1}}(\lambda s)\right)}{\frac{\partial}{\partial s} L_{N_{1}}\left(-\ln L_{X_{1}}(\lambda s)\right)} .
$$

Let $N_{1} \leq_{d-p L t-r} N_{2}$, then, right side of

is decreasing in $s>0$, so, $\frac{\frac{\partial}{\partial s} L_{X_{1}+\ldots+X_{N_{2}}}(\lambda s)}{\frac{d}{d s} L_{X_{1}+\ldots+X_{N_{1}}}(s)}$ is decreasing in $s>0$, therefore the proof is completed. Now, if $\sum_{i=1}^{N_{1}} X_{i} \leq_{d-p L t-r} \sum_{i=1}^{N_{2}} X_{i}$, then the left side of (12) is decreasing in $s>0$, so, $N_{1} \leq_{d-p L t-r} N_{2}$.

Theorem 4.5. Suppose that $X_{i}$ and $Y_{i}$ be sequences of the iid non-negative absolutely continuous random variables that $X_{i} \leq_{d-p L t-r} Y_{i}$ and $X_{i} \leq_{p L t-r} Y_{i}$, for all $i=1,2, \ldots$ and $N_{1}$ and $N_{2}$ be two integer-valued random variables that are independent of $X_{i}$ 's. If $N_{1} \leq_{d-p L t-r} N_{2}$ and $\frac{L_{X_{i}}(s)}{L_{X_{i}}(\lambda s)}, \quad \forall i=1,2, \ldots$ is decreasing in $s>0$, then, $\sum_{i=1}^{N_{1}} X_{i} \leq_{d-p L t-r} \sum_{i=1}^{N_{2}} Y_{i}$.

Proof Using Definitions 3.1 and 4.3, it follows that $\frac{L_{Y_{i}}(\lambda s)}{L_{X_{i}}(s)}$ and $\frac{L_{Y_{i}}^{\prime}(\lambda s)}{L_{X_{i}}^{\prime}(s)}, i=1,2, \ldots$ are decreasing in $s>0$, so,

$$
\frac{L_{Y_{1}+\ldots+Y_{n}}^{\prime}(\lambda s)}{L_{X_{1}+\ldots+X_{n}}^{\prime}(s)}=\frac{n \lambda L_{Y_{1}}^{n-1}(\lambda s) L_{Y_{1}}^{\prime}(\lambda s)}{n L_{X_{1}}^{n-1}(s) L_{X_{1}}^{\prime}(s)}
$$

is decreasing in $s>0$, then, $\sum_{i=1}^{n} X_{i} \leq_{d-p L t-r} \sum_{i=1}^{n} Y_{i}, \forall n>0$. By conditional probability for each $0<s_{1}<s_{2}$, we have

$$
L_{X_{1}+\ldots+X_{N_{2}}}^{\prime}\left(s_{1}\right) L_{Y_{1}+\ldots+Y_{N_{2}}}^{\prime}\left(\lambda s_{2}\right)-L_{X_{1}+\ldots+X_{N_{2}}}^{\prime}\left(s_{2}\right) L_{Y_{1}+\ldots+Y_{N_{2}}}^{\prime}\left(\lambda s_{1}\right) \leq 0,
$$

thus,

$$
\frac{L_{Y_{1}+\ldots+Y_{N_{2}}}^{\prime}(\lambda s)}{L_{X_{1}+\ldots+X_{N_{2}}}^{\prime}(s)}
$$


is decreasing in $s>0$, so, $\sum_{i=1}^{N_{2}} X_{i} \leq_{d-p L t-r} \sum_{i=1}^{N_{2}} Y_{i}$. Since $N_{1} \leq_{d-p L t-r} N_{2}$, then, applying Theorem 4.4, we have $\sum_{i=1}^{N_{1}} X_{i} \leq_{d-p L t-r} \sum_{i=1}^{N_{2}} X_{i} \leq_{d-p L t-r} \sum_{i=1}^{N_{2}} Y_{i}$. Also, decreasing of $\frac{L_{Y_{i}}(\lambda s)}{L_{X_{i}}(s)}$ and $\frac{L_{Y_{i}}^{\prime}(\lambda s)}{L_{X_{i}}^{\prime}(s)}, i=1,2, \ldots$ lead to $\sum_{i=1}^{N_{1}} X_{i} \leq_{d-p L t-r} \sum_{i=1}^{N_{2}} Y_{i}$. If $X_{j} \stackrel{d}{\rightarrow} X$, then $\int_{0}^{\infty} u e^{-s u} f_{i}(u) d u \stackrel{i \rightarrow \infty}{\rightarrow} \int_{0}^{\infty} u e^{-s u} f(u) d u$. Similarly, if $Y_{j} \stackrel{d}{\rightarrow} Y$, then $\int_{0}^{\infty} u e^{-s u} g_{i}(u) d u \stackrel{i \rightarrow \infty}{\rightarrow} \int_{0}^{\infty} u e^{-s u} g(u) d u$. Now, hence

$$
\frac{L_{Y_{i}}^{\prime}(\lambda s)}{L_{X_{i}}^{\prime}(s)} \stackrel{i \rightarrow \infty}{\rightarrow} \frac{L_{Y}^{\prime}(\lambda s)}{L_{X}^{\prime}(s)}
$$

, if $X_{i} \leq_{d-p L t-r} Y_{i}, \quad i=1,2, \ldots$ and $i \longrightarrow \infty$ then, $X \leq_{d-p L t-r} Y$.

\section{PROPORTIONAL LAPLACE TRANSFORM ORDERS FOR WEIGHTED DISTRI- BUTIONS}

Weighted distributions firstly proposed by Fisher (1934) and formulated later by Rao (1965). Statistical applications of the weighted distributions especially to the analysis of data relating to human populations, ecology, forestry and reliability. Readers may refer to Patil and Rao (1977), Jain et al. (1989), Bartoszewicz and Skolimowska (2004) and Belzunce et al. (2004) for more details. Suppose that $X^{w}$ and $Y^{w}$ be weighted versions of the random variables $X$ and $Y$ with density functions $\frac{w(x) f(x)}{E(w(X))}$ and $\frac{w(x) g(x)}{E(w(X))}$ respectively, so

$$
L_{X^{w}}(s)=\int_{0}^{\infty} e^{-s u} \frac{w(u) f(u)}{E(w(U))} d u,
$$

and

$$
L_{Y^{w}}(s)=\int_{0}^{\infty} e^{-s u} \frac{w(u) g(u)}{E(w(U))} d u .
$$

It is clear that $L_{X^{w}}(s)$ and $L_{Y^{w}}(s)$ are decreasing in $s>0$. By definition of the Laplace transform order if $\int_{0}^{\infty} e^{-s u} w(u) f(u) d u \geq \int_{0}^{\infty} e^{-s u} w(u) g(u) d u$ then, $X^{w} \leq_{L t} Y^{w}$.

Here, we consider some special weights:

(1) If $w(x)=e^{l x}$, then,

$$
L_{X} w(s)=\int_{0}^{\infty} \frac{e^{-s u} e^{l u} f(u)}{E\left(e^{l U}\right)} d u=\frac{1}{E\left(e^{l U}\right)} \int_{0}^{\infty} e^{-u(s-l)} f(u) d u=\frac{1}{E\left(e^{l U}\right)} L_{X}(s-l),
$$

so,

$$
L_{X} w(s) \geq L_{Y} w(s) \Leftrightarrow L_{X}(s-l) \geq L_{Y}(s-l)
$$

but,

$$
L_{X^{w}}^{*}(s)=\int_{0}^{\infty} e^{-s u} \frac{w(u) F_{X}(u)}{E(w(U))} d u
$$


and

$$
L_{Y^{w}}^{*}(s)=\int_{0}^{\infty} e^{-s u} \frac{w(u) G_{Y}(u)}{E(w(U))} d u,
$$

and we know, $X^{w} \leq_{L t} Y^{w} \Leftrightarrow L_{X}^{*}(s) \geq L_{Y w}^{*}(s)$.

(2) If $\frac{w(x)}{E w(X)}=\frac{1}{F(x)}$ then,

$$
L_{X^{w}}^{*}(s)=\int_{0}^{\infty} e^{-s u} d u=\frac{1}{s},
$$

so, $X \leq_{L t} X^{w}$ if $L_{X}^{*}(s) \geq \frac{1}{s}$.

(3) We know $L_{X^{w}}^{* *}(s)=\int_{0}^{\infty} e^{-s u} \frac{w(u) \bar{F}_{X}(u)}{E(w(U))} d u$, so, if consider weight function

$$
\frac{w(x)}{E w(X)}=\frac{1}{\bar{F}(x)}
$$

, then, by $X^{w} \leq_{L t} Y^{w} \Leftrightarrow L_{X^{w}}^{* *}(s) \leq L_{Y^{w}}^{* *}(s)$ have,

$$
L_{X^{w}}^{* *}(s)=\int_{0}^{\infty} e^{-s u} \frac{w(u) \bar{F}_{X}(u)}{E(w(U))} d u=\int_{0}^{\infty} e^{-s u} \frac{1}{\bar{F}_{X}(u)} \bar{F}_{X}(u) d u=\frac{1}{s},
$$

so, if $L_{X}^{* *}(s) \leq \frac{1}{s}$ then $X^{w} \leq_{L t} X$.

\section{CONCLUSION}

We recalled some of the stochastic orders that are based on the Laplace transforms and so extended them to proportional state similar to proportional likelihood ratio order and obtained some of their properties and relationships with other orderings. Finally, we studied the proportional Laplace transform order in view of the weighted distributions and some special weights.

\section{REFERENCES}

Alzaid, A., Kim, J. S. and Proschan, F. (1991). Laplace ordering and its applications. Journal of Applied Probability, 28, 116-130.

Bartoszewicz, J. (1999). Characterizations of stochastic orders based on ratios of Laplace transform. Statistics and Probability Letters, 42, 207-212.

Bartoszewicz, J. (2000). Stochastic orders based on the Laplace transform and infinitely divisible distributions. Statistics and Probability Letters, 50, 121-129.

Bartoszewicz, J. and Skolimowska, M. (2004). Stochastic ordering of weighted distributions. University of Wroclaw.

Belzunce, F., Navarro, J. and Del Agula, Y. (2004). Some results on residual entropy function. Metrika, 59, 147-161.

Belzunce, F., Ruiz, J. M. and Ruiz, C. (2002). On preservation of some shifted and proportional orders by systems. Statistics and Probability Letters, 60, 141-154.

Feller, W. (1971). An Introduction to Probability Theory and its Applications. Vol. II., Wiley, New York.

Fisher, R. A. (1934). The effects of methods of ascertainment upon the estimation of frequencies. Annals Eugenics, 6, 13-25. 
Jain, K., Singh, H. and Bagai, I. (1989). Relations for reliability measures of weighted distributions. Communications in Statistics-Theory and Methods, 18, 4393-4412.

Karlin, S. (1968). Total Positivity. Vol. I., Stanford University Press, Stanford, CA.

Klefsjo, B. (1983). A useful ageing property based on the Laplace transform. Journal of Applied Probability, 20, 615-626.

Li, X., Ling, X. and Li, P. (2009). A new stochastic order based upon Laplace transform with applications. Statistical Planning and Inference, 139, 2624-2630.

Nanda, A. K. (2000). Generalized aging classes in terms of Laplace transform. Sankhya, 62, Series A, 258-266.

Patil, G. P. and Rao, C. R. (1997). The weighted distributions: A survey and their applications. Applications of Statistics, Amsterdam: North Holland, 383-405.

Ramos-Romero, H. M. and Sordo-Diaz, M. A. (2001). The proportional likelihood ratio and applications. Questiio, 25, 211-223.

Rao, C. R. (1965). On discrete distributions arising out of methods of ascertainment. Pergamon Press, Oxford and Statistical Publishing Society, 320-332.

Rolski, T. and Stoyan, D. (1976). On the comparison of waiting times in GI/G/1 queues. Operations Research, 24, 197-199.

Shaked, M. and Shanthikumar, J. G. (2007). Stochastic Orders. Academic Press, New York.

Shaked, M. and Wong, T. (1997). Stochastic orders based on ratios of Laplace transforms. Journal of Applied Probability, 34, 404-419.

Jarrahiferiz, J., Mohtashami Borzadaran, G. R. AND Rezaei Roknabadi, A. H.

Department of Statistics

School of Mathematical Sciences

Ferdowsi University of Mashhad

Mashhad-IRAN

Email : grmohtashami@um.ac.ir

Received September 2013 